\title{
SOME MODEL THEORY OF COMPACT LIE GROUPS
}

\author{
ALI NESIN AND ANAND PILLAY
}

\begin{abstract}
We consider questions of first order definability in a compact Lie group $G$. Our main result is that if such $G$ is simple (and centerless) then the Lie group structure of $G$ is first order definable from the abstract group structure. Along the way we also show (i) if $G$ is non-Abelian and connected then a copy of the field $\mathbb{R}$ is interpretable. in $(G, \cdot)$, and (ii) any "1-dimensional" field interpretable in $(\mathbb{R},+, \cdot)$ is definably (i.e., semialgebraically) isomorphic to the ground field $\mathbb{R}$.
\end{abstract}

\section{INTRODUCTION}

It was observed a long time ago (Cartan, van der Waerden) that if $G$ is a compact (semi)simple Lie group, then the Lie group structure of $G$ is "implicitly" defined from the abstract group structure, i.e., any abstract automorphism of $G$ is a homeomorphism (and is thus analytic). We show here that the Lie group structure is "explicitly" defined from the abstract group structure, in a strong sense which we will explain in this introduction. (Note that a "simple" proof of Cartan's theorem is given in [Pi 2].)

We really deal here with Nash groups, namely Lie groups which are definable in $(\mathbb{R},+, \cdot)$ (see $\S 1)$. On the other hand it is known (Chevalley [C]) that any compact Lie group is Lie isomorphic to a real algebraic group which is of course definable in $(\mathbb{R},+, \cdot)$. When we speak of a compact Lie group, we always assume it lives as a definable object in $(\mathbb{R},+, \cdot)$. Given such a group $G$, let $G^{*}$ denote $G$ equipped with all its structure induced from $(\mathbb{R},+, \cdot)$. So the definable sets in $G^{*}$ are the traces in $G$ of the definable set in $(\mathbb{R},+, \cdot)$. We prove

Theorem 0.1. (i) Let $G$ be a compact Lie group which is simple (and centerless). We assume $G$ is a Nash group (so definable in $(\mathbb{R},+, \cdot)$ ). Then there is an isomorphic copy $\left(\mathbb{R}_{1},+, \cdot\right)$ of the real field interpretable in $(G, \cdot)$, there is a Nash group $G_{1}$ over $\mathbb{R}_{1}$ and a definable $($ in $(G, \cdot))$ isomorphism of $G$ with $G_{1}$. Moreover, if $u$ is the unique isomorphism of $\mathbb{R}_{1}$ with $\mathbb{R}$, then $u\left(G_{1}\right)$ and $G$ are Nash isomorphic.

Received by the editors September 12, 1988 and, in revised form, June 26, 1989.

1980 Mathematics Subject Classification (1985 Revision). Primary 03C60; Secondary 22E15.

The second author was supported by NSF grant DMS 8601289 and a fellowship from the Alexander von Humboldt Foundation. 
(ii) Under the assumptions of $(\mathrm{i}),(G, \cdot)=G^{*}$, i.e., every definable set in $G^{*}$ is definable in $(G, \cdot)$.

Our proof follows the proof of the analogous result by Poizat $[\mathrm{Po}, 4 \mathrm{~g}]$ for algebraic groups over algebraically closed fields (stemming from a paper of Zilber $[Z])$. The main problem is just to see that various parts of the proof also hold in the real case.

In $\S 1$ we show that a copy of $\mathbb{R}$ is interpretable in the pure group structure of compact non-Abelian Lie group in a special way. We depend on general properties of compact Lie groups (mainly facts about tori), semialgebraic dimension theory and the first author's observation $[\mathrm{N}]$ that a field can be found in a group resembling $\mathrm{SO}_{3}(\mathbb{R})$. We are somewhat arbitrary in our choice of what general facts about compact Lie groups we assume. Of course, the more of the structure theory of compact Lie groups we assume, the more our proof tends towards a proof by inspection. However we assume nothing about roots and weights. (A separate issue would be to derive all the structural properties of (Nash) Lie groups by real algebraic-geometric (model-theoretic) methods. Some elementary things of this kind were done in [Pi 2] but this is not the purpose of the present paper.)

In $\S 2$ we prove that a "1-dimensional" field interpretable in $(\mathbb{R},+, \cdot)$ is definably isomorphic to $(\mathbb{R},+, \cdot)$. (This amounts to showing that a 1-dimensional Nash field is Nash isomorphic to $\mathbb{R}$.)

In $\S 3$ we show that if $G$ is simple and centerless and $K$ is the field found in $\S 1$, then $G$ is definably interpretable in $K$ (with its induced structure from $(G, \cdot))$, in other words $G$ is contained in the definable closure of $K$, computed in the structure $(G, \cdot)$. Theorem 0.1 follows from the above, together with a result from [Pi 1]: a group interpretable in $(\mathbb{R},+, \cdot)$ is definably isomorphic to a Nash group.

\section{FINDING THE FIELD IN THE GROUP}

To clarify matters we first recall various categories of groups with "real" structure:

A Lie group is an analytic manifold equipped with analytic group structure.

A Nash manifold [S] is an analytic manifold together with a covering by finitely many open sets and analytic isomorphisms from these open sets onto open semialgebraic subsets of $\mathbb{R}^{n}$, with semialgebraic transition maps. A Nash group is a Nash manifold with Nash (analytic semialgebraic) group structure.

A (linear) real algebraic group $G$ is a Zariski closed subgroup of some $\mathrm{GL}_{n}(\mathbb{R})$. Such a group is also a Nash group, as is any open subgroup of $G$.

These are not full subcategories; for example, two Nash groups may be Lie (analytically) isomorphic without being Nash isomorphic.

Now a Nash group $G$ is a real algebraic-geometric object (in particular it is definable in $(\mathbb{R},+, \cdot)$, together with the Nash group structure). We thus have (see [Pi 1]) 
Fact 1.1. Let $G$ be a Nash group. Then

(i) any semialgebraic (i.e., definable in $(\mathbb{R},+, \cdot)$ ) subgroup of $G$ is closed.

(ii) $G$ has the DCC on semialgebraic subgroups.

Now any semialgebraic set $X \subseteq \mathbb{R}^{n}$ has a dimension, $\operatorname{dim} X$ which can be defined in a number of ways (see, e.g. [S]); we have

Fact 1.2.

(i) Let $X$ be semialgebraic. Then $\operatorname{dim} X=$ algebraic-geometrical dimension of the Zariski closure of $X$.

(ii) If $G$ is a Nash group, then $\operatorname{dim} G=\operatorname{dimension}$ of $G$ as a manifold.

(iii) Let $G$ be a Nash group (or manifold) and $X$ a semialgebraic subset of $G$. Then $\operatorname{dim} X=\operatorname{dim} G \Rightarrow X$ contains a nonempty open subset of $G$.

If $\bar{a} \in \mathbb{R}^{n}$ and $A \subseteq \mathbb{R}$, then $\operatorname{dim}(\bar{a} / A)$ is defined to be the maximum of $\left\{\operatorname{dim} X: X \subseteq \mathbb{R}^{n}\right.$ is semialgebraic, defined with parameters in $A$ and $\left.\bar{a} \in X\right\}$.

Fact 1.3.

(i) If $\bar{a} \in \mathbb{R}^{n}, \bar{b} \in \mathbb{R}^{n}, A \subseteq \mathbb{R}$ and $\bar{a} \in \operatorname{acl}(\bar{b} A)$ (where acl is computed in $(\mathbb{R},+, \cdot)$ then $\operatorname{dim}(\bar{a} / A) \leq \operatorname{dim}(\bar{b} / A)$.

(ii) If $X \subseteq \mathbb{R}^{n}$ is semialgebraic and defined over $A \subseteq \mathbb{R}$, then there is an $\bar{a} \in X$ with $\operatorname{dim}(\bar{a} / A)=\operatorname{dim} X$.

We should also mention a fact which can be easily deduced from results in [Pi 1].

Fact 1.4. Let $G$ be a Nash group and $H$ a semialgebraic normal subgroup of $G$. Then there is a Nash group $K$ and a semialgebraic isomorphism of $G / H$ with $K$, such that the manifold topology on $K$ corresponds to the quotient topology on $G / H$.

We now recall some general facts about compact Lie groups.

Fact 1.5 (Chevalley [C]). Any compact Lie group is isomorphic as a Lie group to a real algebraic group.

From now on, $G$ denotes a compact connected Lie group. $T^{1}$ denotes the torus $\mathbb{R} / \mathbb{Z}$ and $T^{k}=T^{1} \times \cdots \times T^{1}$ ( $k$ times $)$.

Fact 1.6 [Bou]. If $G$ is Abelian then $G$ is isomorphic (as a Lie group) to some $T^{k}$.

Definition 1.7. A maximal torus of $G$ is a maximal closed connected Abelian subgroup of $G$.

Fact 1.8 [Bou].

(i) Any maximal torus of $G$ is a maximal Abelian subgroup of $G$.

(ii) $G$ is covered by its maximal tori, which are, moreover, all conjugate in $G$. 
Fact 1.9 [Bou]. The derived group $G^{\prime}$ of $G$ has a finite center. Moreover, $G$ is the almost direct product $Z(G) \cdot G^{\prime}$ (i.e., $G=Z(G) \cdot G^{\prime}$ and $Z(G) \cap G^{\prime}$ is finite).

Before stating and proving the main result of this section we first observe that many of the data mentioned above are definable in the pure group language. We assume $G$ is connected unless otherwise stated. Any other groups mentioned are also Lie groups.

Lemma 1.10. Any maximal torus of $G$ is definable in $(G, \cdot)$.

Proof. Note first that, by Fact 1.1(ii), for any $A \subset G$ there is finite $A_{0} \subset G$ such that $C_{G}(A)=C_{G}\left(A_{0}\right)$. Thus, if $T$ is a maximal torus of $G$ then $Z\left(C_{G}(T)\right)$ is definable in $(G, \cdot)$ and is equal to $T$ by Fact 1.8(i).

Lemma 1.11. Let $A$ be compact Abelian (not necessarily connected). Then $A^{\circ}$, the connected component of $A$, is definable in $(A, \cdot)$.

Proof. By Fact $1.6, A^{\circ}$ is divisible and clearly $\left|A / A^{\circ}\right|<\omega$. Thus, for some $n, A^{\circ}=n \cdot A$, and so $A^{\circ}$ is definable in $(A, \cdot)$.

Lemma 1.12. Let $H$ be compact, not necessarily connected. Then $H^{\circ}$, the connected component of $H$, is definable in $(H, \cdot)$.

Proof. Clearly $H^{\circ}$ has finite index in $H$. For each maximal torus $T$ of $H^{\circ}$, $Z\left(C_{H}(T)\right)$ is an Abelian definable subgroup of $H$ in which $T$ has finite index. As in Lemma 1.11, $T$ is definable in $(H, \cdot)$ By 1.8(ii), $H^{\circ}=\bigcup_{h \in H} T^{h}$ which is thus definable in $(H, \cdot)$.

Lemma 1.13. The derived group $G^{\prime}$ of $G$ is definable in $(G, \cdot)$.

Proof. By [Pi 2] and Fact 1.5, $G^{\prime}$ is semialgebraic (so closed), and, moreover, there are $a_{1}, \ldots, a_{n} \in G$ such that $\left(a_{1}^{G} \cdot a_{1}^{-1}\right) \cdots\left(a_{n}^{G} \cdot a_{n}^{-1}\right)$ contains an open subset of $G^{\prime}$. Since $G^{\prime}$ is also compact, finitely many translates of this set cover $G^{\prime}$, whereby $G^{\prime}$ is definable in $(G, \cdot)$.

We now state the main result of this section.

Proposition 1.14. Let $G$ be a compact connected non-Abelian Lie group. Then there are, definable just in the group language, $X \subset G$, an equivalence relation $E$ on $X$ with finite classes, and a field structure on $X / E$. Moreover, $X$ (and so $X / E$ ) is 1-dimensional (as a semialgebraic set).

Proof. Let $H$ be a minimal connected non-Abelian subgroup of $G$ definable (in $(G, \cdot)$ ) (where connected means topologically). By Fact $1.9 H=H^{\prime}$ has finite center. Moreover, $H / Z(H)$ is centerless $(H / Z(H))^{\prime}=H / Z(H)$ and thus, by Fact 1.9, $H / Z(H)$ has finite center, the preimage of which in $H$ is finite, normal and thus central). Clearly, every proper connected definable (in $(G, \cdot))$ subgroup of $H / Z(H)$ is Abelian (the same being true of $H$ ). Let us put $H_{1}=H / Z(H)$. So $H_{1}$ is a compact connected non-Abelian centerless Lie group, every definable (in $\left.H_{1}, \cdot\right)$ ) proper connected subgroup of which is Abelian. We now work in $H_{1}$. 
Claim 1. Every nontrivial element $a \in H_{1}$ is in a unique maximal torus $T$ which equals $C^{\circ}(a)$, the connected component of the centralizer of $a$ in $H$.

Proof. Let $a \neq 1, a \in H_{1}$. Let $T$ be a maximal torus containing $a$ (by (1.8(ii)). So $T \subset C(a)$. As $C^{\circ}(a)$ has finite index in $C(a)$ and $T$ is connected, $T \subset C^{\circ}(a)$, and as $a$ is not central, $C^{\circ}(a) \varsubsetneqq H_{1}$. So $C^{\circ}(a)$ is Abelian, thus equals $T$.

For $a \neq 1, a \in H_{1}$, we let $T(a)$ denote the unique maximal torus containing a. $T$ is an arbitrary maximal torus (of $H_{1}$ ). Notice that, by Claim 1 , tori are disjoint.

Claim 2. $C(T)=T$ and $T$ has finite index in $N(T)$.

Proof. By 1.8(ii), $T$ is not normal in $H_{1}$, so $N(T) \supsetneqq H_{1},(N(T))^{\circ}$ contains $T$ and is Abelian. Thus $N(T)^{\circ}=T$, hence $[N(T): T]$ is finite. If $T \neq C(T)$, let $a \in C(T)-T$. Then, as $T$ is connected, $T \subset C^{\circ}(a)=T(a)$. Thus $T=T(a)$. So $a \in T$, a contradiction.

Claim 3. $N(T)-T$ contains an involution. Moreover, any involution of $N(T)-T$ acts on $T$ as $x \rightarrow x^{-1}$.

Proof. By Facts 1.6 and 1.8, $H_{1}$ contains involutions $i, j$ such that $T(i) \neq$ $T(j)$. Now $(i j)^{i}=(i j)^{j}=j i=(i j)^{-1}$, so, by Claim 1, both $i$ and $j$ normalize $T(i j)$. If $i \in T(i j)$ then also $j \in T(i j)$, whereby $T(i)=T(i j)=T(j)$, which is impossible. So $i \in N(T(i j))-T(i j)$. Without loss $T(i j)=T$ (by Fact $1.8(\mathrm{ii}))$.

Now let $a \in N(T)-T$ be an involution. Let $A=C(a) \cap T$ and $B=\{x \in$ $\left.T: x^{a}=x^{-1}\right\} . A$ and $B$ are subgroups of $T$. Moreover, as $T$ is divisible, $T=T^{2}$ and every element of $T$ can be written in the form $t t^{a} t\left(t^{a}\right)^{-1}$. But $t t^{a} \in A$ and $t\left(t^{a}\right)^{-1} \in B$. Thus $T=A B$. But $C(a) \cap T$ is finite, for, otherwise, clearly $T(a)=T$, which is impossible. Thus $B$ (which is definable) has finite index in $T$. So $B=T$ and Claim 3 is proved.

Claim 4. $T$ has a unique involution, $\operatorname{dim} T=1$ and $|N(T) / T|=2$.

Proof. Let $i, j$ be involutions of $T$. Let $a \in N(T)-T$ be an involution (by Claim 3). By Claim 3 again, $i^{a}=i, j^{a}=j$. Thus both $i$ and $j$ are in $N(T(a))$. By Claim 3 again $i j \in C(T(a))=T(a) \neq T$. But $i j \in T$. Thus $i j=1, i=j$. So $T$ has a unique involution. As $T$ is a torus, its dimension (as a manifold or as semialgebraic set) must be 1 . Since the only nontrivial Lie automorphism of $T^{1}$ is $x \rightarrow x^{-1}$, it follows that $|N(T) / T|=2$.

At this point (in fact earlier) it is known that $H_{1}$ is $\mathrm{SO}_{3}(\mathbb{R})$. In any case, as in [N], we easily find a projective plane on the set of involutions $I$ of $H_{1}$. The lines are sets of the form $N(T)-T$ for $T$ a maximal torus. If $T=T(i)$ we call such a line $L(i)$. One easily checks from the above that $L(i j)$ is the unique line containing $i$ and $j$ and that $k$ is the unique point in $L(i) \cap L(j)$, 
where $k$ is the unique involution of $T(i j)$. By Bachman [B] this projective plane is the projective plane of a field $K$ which is thus definable on any line $N(T)-T$, minus a point. Thus $\operatorname{dim} K=1$ (as a semialgebraic set). Noting that $H_{1}$ arose from $G$ as the quotient of a definable (in $(G, \cdot)$ ) subgroup by its finite center, we see that Proposition 1.14 is proved.

\section{Classifying SEMIALgebraic FieldS}

In this section we prove

Theorem 2.1. Let $K$ be a field interpretable in $(\mathbb{R},+, \cdot)$ such that $\operatorname{dim} K=1$. Then there is an isomorphism of $K$ with $\mathbb{R}$ that is definable in $(\mathbb{R},+, \cdot)$.

Remark. $(\mathbb{R},+, \cdot)$ has so-called elimination of imaginaries, which amounts to saying that, for any definable $X \subseteq \mathbb{R}^{n}$ and definable equivalence relation $E$ on $X$, there is a definable $Y \subseteq \mathbb{R}^{n}$ and a definable bijection $f: X / E \rightarrow Y$. This enables us to talk about $\operatorname{dim} X / E$, as definable bijections preserve dimension.

We will need the following fact, pointed out to us by $L$. van den Dries.

Lemma 2.2. Let $G$ be a Nash group, with $\operatorname{dim} G=n$. Then the adjoint representation Ad: $G \rightarrow \mathrm{GL}_{n}(\mathbb{R})$ is semialgebraic.

Outline of proof. Identify semialgebraically an open neighborhood of 1 in $G$ with an open subset of $\mathbb{R}^{n}$. For $x \in G$, Int $x$ is the map $g \rightarrow g^{x}$ and $\operatorname{Ad} x$ is the Jacobian matrix of Int $x$ at 1 . As $G$ is covered by a finite number of open semialgebraic sets, Ad $x$ is uniformly semialgebraic in $x$, which proves the lemma.

Corollary 2.3. Let $G$ be a centerless group, interpretable in $(\mathbb{R},+, \cdot)$. Then there is a semialgebraic isomorphism of $G$ with a subgroup of some $\mathrm{GL}_{n}(\mathbb{R})$.

Proof. By [Pi 2], $G$ can be semialgebraically equipped with Nash group structure, i.e., $G$ has a semialgebraic covering by a finite number of open semialgebraic subsets of $\mathbb{R}^{n}$ (where $n=\operatorname{dim} G$ ) such that the transition maps are Nash and group multiplication is Nash. Namely, we can identify $G$ semialgebraically with a Nash group. Now the kernel of $\operatorname{Ad}$ is $Z(G)$. As $G$ is centerless, by Lemma 2.2 , we see $G$ is semialgebraically isomorphic to a (semialgebraic) subgroup of $\mathrm{GL}_{n}(\mathbb{R})$.

We are now set for the proof of Theorem 2.1. The main point is to show that $(K,+)$ is semialgebraically isomorphic to $(\mathbb{R},+)$. (Note that by [Pi 1], $K$ is real closed and in fact by [Pon], is abstractly isomorphic to $\mathbb{R}$.) Let $K^{+}$be the additive group of $K$ and $K_{+}^{*}$ the "positive" elements of the multiplicative group of $K$. Both $K^{+}$and $K_{+}^{*}$ have no proper subgroups of finite index. Thus also the centerless group $K^{+} \rtimes K_{+}^{*}$ (where $K_{+}^{*}$ acts on $K^{+}$by multiplication), which is also interpretable in $(\mathbb{R},+, \cdot)$, has no proper subgroups of finite index. By Corollary 2.3, $K^{+} \rtimes K_{+}^{*}$ is semialgebraically isomorphic to a semialgebraic linear group $G . G$ is clearly connected as a Lie group. Let $H$ be the image 
of $K^{+}$in $G$. By [Pi 2], $G$ has finite index in a real algebraic (linear) group $G_{1}$ which is connected as an algebraic group. Let $H_{1}$ be the Zariski closure of $H$ in $G_{1}$. Then, by Fact 1.2, the algebraic-geometrical dimension of $H_{1}$ is 1 . $H_{1}$ is also connected as an algebraic group (because $H$ has no subgroups of finite index). Let now $\left(G_{1}\right)_{\mathbb{C}}$ be the complexification of $G_{1}$, i.e., the smallest complex algebraic group containing $G_{1}$, and similarly for $\left(H_{1}\right)_{\mathbb{C}}$, with $\left(H_{1}\right)_{\mathbb{C}}<$ $\left(G_{1}\right)_{\mathbb{C}}$. Both $\left(H_{1}\right)_{\mathbb{C}},\left(G_{1}\right)_{\mathbb{C}}$ are by [B1] connected algebraic groups, defined over $\mathbb{R}$, and, moreover, the real points of $\left(H_{1}\right)_{\mathbb{C}},\left(G_{1}\right)_{\mathbb{C}}$ are $H_{1}, G_{1}$, respectively. Furthermore, $\left(H_{1}\right)_{\mathbb{C}}$ is 1-dimensional as an algebraic (and also the dimension of $\left(G_{1}\right)_{\mathbb{C}}$ is 2). It is easy to see that $\left(H_{1}\right)_{\mathbb{C}}$ is normal in $\left(G_{1}\right)_{\mathbb{C}}$ (if $g \in G$ then $g$ normalizes $H$, so $\left(H_{1}\right)_{\mathbb{C}}^{g} \cap\left(H_{1}\right)_{\mathbb{C}}$ is infinite and equals $\left(H_{1}\right)_{\mathbb{C}}$ as the latter is connected. The normalizer of $\left(H_{1}\right)_{\mathbb{C}}$ in $\left(G_{1}\right)_{\mathbb{C}}$ is algebraic and contains $G$, so must be all of $\left.\left(G_{1}\right)_{\mathbb{C}}\right)$. As $H$ is not central in $G,\left(H_{1}\right)_{\mathbb{C}}$ is not central in $\left(G_{1}\right)_{\mathbb{C}}$. Thus

An infinite algebraic group acts faithfully on $\left(H_{1}\right)_{\mathbb{C}}$.

Now, as $\left(H_{1}\right)_{\mathbb{C}}$ is a 1-dimensional connected complex algebraic group, it is $[B 2,10.9]$ isomorphic as an algebraic group to $\mathbb{C}^{+}$or to $\mathbb{C}^{*}$. As $\mathbb{C}^{*}$ does not have an infinite group of automorphisms, by $(*)$, it must be $\mathbb{C}^{+}$. By [B2, 10.9], as $\left(H_{1}\right)_{\mathbb{C}}$ is defined over $\mathbb{R}$, the isomorphism of $\left(H_{1}\right)_{\mathbb{C}}$ with $\mathbb{C}^{+}$(which is a polynomial map $f$, say) can be taken to be defined over $\mathbb{R}$. But then $f$ takes the real points of $\left(H_{1}\right)_{\mathbb{C}}$ to the real points of $\mathbb{C}^{+}$. Namely, $H_{1}$ and $\mathbb{R}^{+}$are isomorphic by a polynomial map in $\mathbb{R}$. As $\mathbb{R}^{+}$has no subgroup of finite index, the same is true for $H_{1}$. Then $H=H_{1}$ and we see that $K^{+}$is semialgebraically isomorphic to $\mathbb{R}^{+}$.

Let us now identify $K^{+}$with $\mathbb{R}^{+}$. First note that any definable (in $(\mathbb{R},+, \cdot)$ ) endomorphism of $\mathbb{R}^{+}$is linear: for if $f$ is such, then $R=\{a \in \mathbb{R}: f(a \cdot x)=$ $a \cdot f(x) \forall x \in \mathbb{R}\}$ is a definable infinite subgroup of $\mathbb{R}^{+}$, so equals $\mathbb{R}$.

Now let $a \in \mathbb{R}$ be the 1 of $K$ (so $a \neq 0$ ), and let $\circ$ be the multiplication of $K$. By the above note, $a x y=a x \circ a y$, and so the map $x \rightarrow a x$ is a 1-1 onto map $\mathbb{R} \rightarrow K$, which is an isomorphism of $(\mathbb{R},+, \cdot)$ with the field $K$. We have shown that $K$ is definably isomorphic to $(\mathbb{R},+, \cdot)$.

\section{FINDING THE GROUP IN THE FIELD}

We begin with some definitions. Let $M$ be an $L$-structure, and $X, Y$ subsets of $M$ (or $\left.M^{\mathrm{eq}}\right)$. We will say $Y \subset_{u} \operatorname{acl}(X)(Y$ is uniformly in the algebraic closure of $X$ ) if there is a formula $\varphi(y, \bar{x})$ of the language $L$ such that, for some $n \in \omega, M \models \forall \bar{x} \exists^{\leq n} y \varphi(y, \bar{x})$, and, for all $b \in Y$, there is $\bar{a} \subset X$ such that $M \models \varphi(b, \bar{a})$. We will say $Y \subset_{u} \operatorname{dcl}(X) \quad(Y$ is uniformly in the definable closure of $X)$ if $\varphi(y, \bar{x})$ can be chosen as above but with $n=1$.

We prove

Theorem 3.1. Let $G$ be a compact simple (centerless) Lie group. Then there is a 1-dimensional field $K$ interpretable in $(G, \cdot)$ and some finite $\bar{c} \subset G$ such that $G \subset_{u} \operatorname{dcl}(K \cup \bar{c})$ in the sense of the structure $(G, \cdot)$. 
We first observe

Lemma 3.2. Under the assumptions of 3.1, $K$ and $\bar{c}$ can be found with $G \subset_{u}$ $\operatorname{acl}(K \cup \bar{c})$.

Proof. Let $K$ be as given by Proposition 1.14 (i.e., $K \subset X / E$ ), where $X \subset G$ is definable in $(G, \cdot)$ and $E$ is a definable equivalence relation with finite classes. Let $Y \subset X$ be the union of the $E$-classes which are members of $K . G$ being real algebraic and simple, we can apply Remark 3.6 of [Pi 2] which implies that there is an open neighborhood of 1 in $G$ and finitely many elements $\bar{c} \subset G$ such that $U \subset_{u} \operatorname{dcl}(Y \cup \bar{c})$. As $G$ is compact, we extend $\bar{c}$ to $\bar{c}^{\prime}$ such that $G \subset_{u} \operatorname{dcl}\left(Y \cup \bar{c}^{\prime}\right)$ computed in $\left.(G, \cdot)\right)$. This proves the lemma.

Proof of Theorem 3.1. We want to use the fact that $G$ has no finite normal subgroups to see that we can add finitely many additional parameters to the $\bar{c}$ from Lemma 3.2 to obtain $\bar{d}$ with $G \subset_{u} \operatorname{dcl}(K \cup \bar{d})$.

First, by Lemma 3.2, we can choose a finite set $\bar{c} \subset G$, a formula $\varphi(y, \bar{x})$ of the pure group language and some $k<\omega$ such that $G \models \forall \bar{x} \exists^{\leq k} y \varphi(y, \bar{x})$, and, for all $b \in G$, there is a $\bar{a} \subset K \cup \bar{c}$ with $G \models \varphi(g, \bar{a})$ and $k$ smallest possible.

Before continuing the proof we introduce some notation. Remember that $G$ is a real algebraic group, thus any subset of $G$ defined in the group language is also a semialgebraic set and therefore has a dimension (Fact 1.2). Suppose $\operatorname{dim} G=n$, and let $\psi(y)$ be a formula in the group language (maybe with parameters). We will say that, for almost all $b \in G, G \models \psi(b)$ if $\{b \in G: G \models$ $\neg \psi(b)\}$ has dimension $<n$, and we observe

Remark 3.3. Either, for almost all $b \in G, G \models \psi(b)$ or $\{b \in G: G \models \neg \psi(b)\}$ contains a nonempty open set.

For $A \subset G$ and $b \in G$ we will say that $b$ is generic over $A$ if $\operatorname{dim}(b / A)=n$.

We now return to our choice of $\varphi$ and $k$. Let $\psi(y, \bar{x})$ be $\varphi(y, \bar{x}) \wedge$ $\exists^{=k} y \varphi(y, \bar{x})$.

Claim 3.4. For almost all $b \in G$ there is an $\bar{a} \subset K \cup \bar{c}$ with $G \models \psi(b, \bar{a})$.

Proof. If not, then, by 3.3, there is a nonempty open set $U \subset G$ such that, for all $b \in U$, there is an $\bar{a} \subset K \cup \bar{c}$ with $G \models \varphi(b, \bar{a}) \wedge \exists^{<k} y \varphi(y, \bar{a})$. As $G$ is compact, finitely many translates of $U$ cover $G$, so, by adding a finite number of parameters to $\bar{c}$, we easily contradict least choice of $k$.

Claim 3.5. For almost all $b \in G$, if $G \models \psi\left(b, \bar{a}_{1}\right) \wedge \psi\left(b, \bar{a}_{2}\right)$, where $\bar{a}_{1}, \bar{a}_{2} \subset$ $K \cup \bar{c}$, then $G \models \forall y\left(\psi\left(y, \bar{a}_{1}\right) \leftrightarrow \psi\left(y, \bar{a}_{2}\right)\right)$.

Proof. Again by using 3.3, we see that, if not, there is a nonempty open set $U \subset G$ such that, for all $b \in U$, there are $\bar{a}_{1}, \bar{a}_{2} \subset K \cup \bar{c}$ such that $G \models$ $\psi\left(b, \bar{a}_{1}\right) \wedge \psi\left(b, \bar{a}_{2}\right)$ and $G \models \neg \forall y\left(\psi\left(y, \bar{a}_{1}\right) \leftrightarrow \psi\left(y, \bar{a}_{2}\right)\right)$. Note that then $G \models$ $\exists^{<k} y\left(\psi\left(y, \bar{a}_{1}\right) \wedge \psi\left(y, \bar{a}_{2}\right)\right)$; so, again by compactness of $G$, we contradict least choice of $k$. 
Let now $A \subset G$ be a finite set including $\bar{c}$ and the parameters defining $K$. Let $Z=\left\{b \in G: \exists \bar{a} \subset K \cup \bar{c}, G \models \psi(b, \bar{a})\right.$, and, $\forall \bar{a}_{1}, \bar{a}_{2} \subset K \cup \bar{c}$, if $G \models$ $\psi\left(b, \bar{a}_{1}\right) \wedge \psi\left(b, \bar{a}_{2}\right)$ then $G \vDash \forall y\left(\psi\left(y, \bar{a}_{1}\right) \leftrightarrow \psi\left(y, \bar{a}_{2}\right)\right\} . Z$ is $A$-definable (in the group language) and, by the previous two claims, $\operatorname{dim}(G-Z)<n$. In particular, note that if $b$ is generic over $A$ then $b \in Z$. We can define an equivalence relation $E$ on $Z$ by $y_{1} E y_{2}$ if $\exists \bar{a} \subset K \cup \bar{c} G \models \psi\left(y_{1}, \bar{a}\right) \wedge \psi\left(y_{2}, \bar{a}\right)$.

Note that $E$ is $A$-definable and, for $b \in Z$, the $E$-class of $b$ has exactly $k$ elements.

Claim 3.6. For all $g \in G$, almost all $b \in Z, " g b \in Z$ and for all $b_{1}, b_{1} E b$ implies $g b_{1} \in Z$ and $b g_{1} E g b "(*)$.

Proof. If not, there is a $g \in G$ and an open set $U$ of $b \in G$ which fail to satisfy $(*)$. Choose $b \in U$ which is generic over $A \cup\{g\}$. Now, for any $b_{1}$, if $b_{1} E b$ then $b \in \operatorname{acl}\left(b_{1} \cup A\right)$ and thus, by Fact $1.3, b_{1}$ is generic over $A \cup\{g\}$. So, by Fact 1.3 again, $g b_{1}$ is generic over $A$ and $g b_{1} \in Z$. So, as (*) fails, there is $b_{1} E b$ with $g b_{1} \in Z$ but $\neg\left(g b_{1} E g b\right)$. Let $\bar{a}_{1} \subset K \cup \bar{c}$ be such that $G \models \psi\left(b, \bar{a}_{1}\right)$, and let $\bar{a}_{2} \subset K \cup \bar{c}$ be such that $G \models \psi\left(g b, \bar{a}_{2}\right)$. Thus the formula $\psi\left(y, \bar{a}_{1}\right) \wedge \psi\left(g y, \bar{a}_{2}\right)$ is satisfied by $b$ but not by $b_{1}$, and hence it is satisfied by $<k$ elements.

As $b$ is generic over $A \cup\{g\}$, there is, by Fact 1.2, an open set $V \subset G$ such that, for all $y \in V$, there are $\bar{x}_{1}, \bar{x}_{2} \subset K \cup \bar{c}$ such that $G \models \psi\left(y, \bar{x}_{1}\right) \wedge$ $\psi\left(g y, \bar{x}_{2}\right)$ and $G \vDash \exists^{<k} y\left(\psi\left(y, \bar{x}_{1}\right) \wedge \psi\left(g y, \bar{x}_{2}\right)\right)$. Again by compactness of $G$, after adding $g$ and finitely many additional parameters to $\bar{c}$, we contradict the least choice of $k$. This proves Claim 3.6.

Now let $L=\{g \in G$ : for almost all $b \in Z$, if $b$ satisfies $(*)$ then $b E g b\}$. It is easy to see that $L$ is a (finite) normal subgroup of $G$, and thus $L=\{1\}$.

We can now conclude the proof of Theorem 3.1. Let $g \in G$ be generic over $A$. Let $\bar{a} \subset K \cup \bar{c}$ be such that $G \models \psi(g, \bar{a})$, and let $g / E=\{g=$ $\left.g_{1}, g_{2}, \ldots, g_{k}\right\}$.

For $i=2, \ldots, k$, let $h_{i}=g^{-1} g_{i}$. As $L=\{1\}$, we can, for each $i=$ $2, \ldots, k$, choose $a_{i}$ generic over $A \cup\left\{g, g_{i}\right\}$ such that $\neg\left(h_{i} a_{i} E a_{i}\right)$. Let $\bar{c}_{i}, \bar{d}_{i}$, for $i=2, \ldots, k$, be such that $G \models \psi\left(a_{i}, \bar{c}_{i}\right) \wedge \psi\left(g a_{i}, \bar{d}_{i}\right), \bar{c}_{i}, \bar{d}_{i} \subset$ $K \cup \bar{c}$. It is then clear that $g \in \operatorname{dcl}\left(\bar{a}, \bar{c}_{2}, \ldots, \bar{c}_{k}, \bar{d}_{2}, \ldots, \bar{d}_{k}\right)$; in fact $g$ is the unique $y \in G$ such that $G \models \psi(y, \bar{a})$ and such that, whenever $G \models \psi\left(a, \bar{c}_{i}\right)$, then also $G \models \psi\left(y a, \bar{d}_{i}\right)$. (Note that $g$ satisfies these conditions. If $g^{\prime}$ does also then $g^{\prime}=g_{i}$, some $i=2, \ldots, k$; but, then $g_{i} a_{i} E g a_{i}$ so $g^{-1} g_{i} a_{i} E a_{i}$, contradicting the choice of $a_{i}$.)

Thus we have a formula $\chi\left(y, \bar{z}_{1}, \bar{z}_{2}\right)$ such that

$$
G \models \forall \bar{z}_{1}, \bar{z}_{2} \exists^{\leq 1} y \chi\left(y, \bar{z}_{1}, \bar{z}_{2}\right)
$$

and

$$
G \models \exists \bar{z}_{1} \subset K \chi\left(g, \bar{z}_{1}, \bar{c}\right) .
$$


As $g$ was generic over $A$, there is an open set $U$ of $g^{\prime}$ such that $G \models$ $\exists \bar{z}_{1} \subset K \chi\left(g^{\prime}, \bar{z}_{1}, \bar{c}\right)$. Again by compactness of $G$, we can add finitely many parameters to $\bar{c}$ to get $\bar{c}^{\prime}$ such that $G \subset_{u} \operatorname{dcl}\left(K \cup \bar{c}^{\prime}\right)$, which proves Theorem 3.1 .

We now prove Theorem 0.1 .

Proof of Theorem 0.1. (i) Let $G$ be our simple centerless compact Lie group which we assume to live in $(\mathbb{R},+, \cdot)$ as a real algebraic group. Let, by Theorem $3.1, K$ be a 1-dimensional field (as a semialgebraic set) which is interpretable in $(G, \cdot)$ such that $G \subset_{u} \operatorname{dcl}(K \cup \bar{c})$ (some $\left.\bar{c} \subset G\right)$ in the sense of $(G, \cdot)$. Easily, $G$ is definably isomorphic (in $(G, \cdot)$ ) to a group $G_{1} \subseteq K^{n} / E$, where $E$ is a definable equivalence relation in $(G, \cdot)$ on $K^{n}$. Now $K$ (being interpretable in $(\mathbb{R},+, \cdot)$ and 1 -dimensional) is, by Theorem 2.1 , definably (in $(\mathbb{R},+, \cdot)$ ) isomorphic to $(\mathbb{R},+, \cdot)$ by an isomorphism $u$, say. So $u\left(G_{1}\right)$ is interpretable in $(\mathbb{R},+, \cdot)$. It follows that $G_{1}$ is interpretable also in $(K,+, \cdot)$. By [Pi 1], $G_{1}$ is definably isomorphic in $(K,+, \cdot)$ to a Nash group $G_{2}$ over $K$. As the operations + and $\cdot$ of $K$ are definable in $(G, \cdot)$ and $G_{1}$ is definably isomorphic (in $(G, \cdot))$ to $(G, \cdot)$, we conclude that a Nash group structure can be found on $(G, \cdot)$ definably in $(G, \cdot)$. That this Nash group structure is the same as the original can be deduced from the fact that $u\left(G_{2}\right)$ and $G$ are definably (in $(\mathbb{R},+, \cdot)$ ) isomorphic and that any definable isomorphism of Nash groups is a Nash isomorphism.

(ii) We have an isomorphism $f: G \rightarrow G_{2}$ definable in $(G, \cdot)$ and an isomorphism $u: G_{2} \rightarrow u\left(G_{2}\right)$ definable in $(\mathbb{R},+, \cdot)$. Let $g=u \circ f$. Let $X$ be a semialgebraic set in $G$. Then $g(X)$ is a semialgebraic set in $u\left(G_{2}\right)$, so $u^{-1} \circ g(X)$ is a semialgebraic set in $G_{2}$ (when we view $G_{2}$ as definable in $K$ ). Now $X=f^{-1}\left(u^{-1} \circ g(X)\right)$. But $f^{-1}$ is definable in $(G, \cdot)$. Thus, also, $X$ is definable in $(G, \cdot)$.

This completes the proof of Theorem 0.1 .

\section{REFERENCES}

[Ba] F. Bachman, Geometric structures, Nauka, Moscow, 1969.

[B1] A. Borel, Groupes algébriques linéaires, Ann. of Math. 2 (1950), 20-82.

[B2] _ Linear algebraic groups, Benjamin, 1969.

[Bou] N. Bourbaki, Groupes et algèbres de Lie, Chapitre 9, Masson, Paris 1982.

[C] C. Chevalley, Theory of Lie groups, Princeton Univ. Press, Princeton, N. J., 1946.

[N] A. Nesin, Nonsolvable groups of Morley rank 3, J. Algebra (1988+) (to appear).

[Pi 1] A. Pillay, Groups and fields definable in 0-minimal structures, J. Pure Appl. Algebra (to appear).

[Pi 2] An application of model theory to real and p-adic algebraic groups, preprint, 1988.

[Po] B. Poizat, Groupes stables, Nur al-Mantiq wal-Marifah, 1987.

[Pon] L. S. Pontriagin, Topological groups, Gordon and Breach, New York, 1966. 
[S] M. Shiota, Nash manifolds, Springer Verlag, Berlin and New York, 1987.

[Z] B. Zil'ber, Some model theory of simple algebraic groups over algebraically closed fields, Colloq. Math. 48 (1984), 173-180.

(Anand Pillay) Department of Mathematics, University of Notre Dame, Notre Dame, INDIANA 46556

Current address, Ali Nesin: Department of Mathematics, University of California, Irvine, California 92717 